\title{
Authors' reply re: Maternal transmission of SARS-COV-2 to the neonate, and possible routes for such transmission: A systematic review and critical analysis. (Response to BJOG-20-1416)
}

\author{
Kate Walker ${ }^{1}$, Keelin ODonoghue ${ }^{2}$, Nicky Grace ${ }^{3}$, Jon Dorling ${ }^{4}$, Jeannette Comeau ${ }^{4}$, \\ Wentao $\mathrm{Li}^{5}$, and James Thornton ${ }^{6}$ \\ ${ }^{1}$ University of Nottingham \\ ${ }^{2}$ University College Cork \\ ${ }^{3}$ University of Nottingham Faculty of Arts \\ ${ }^{4}$ Dalhousie University Faculty of Medicine \\ ${ }^{5}$ Monash University \\ ${ }^{6}$ Nottingham City Hospital
}

July 20, 2020

Authors' reply re: 'Maternal transmission of SARS-COV-2 to the neonate, and possible routes for such transmission: A systematic review and critical analysis (Response to BJOG-20-1416)

Kate F Walker ${ }^{1}$, Keelin O'Donoghue ${ }^{2}$, Nicky Grace ${ }^{3}$, Jon Dorling ${ }^{4}$, Jeannette L Comeau ${ }^{4}$, Wentao Li $^{5}$ Jim $^{\prime}$ G Thornton ${ }^{1}$

${ }^{1}$ Division of Child Health, Obstetrics and Gynaecology, School of Medicine, University of Nottingham

${ }^{2}$ The Irish Centre for Maternal and Child Health, University College Cork, Cork University Maternity Hospital, Cork, Ireland

${ }^{3}$ School of English, University of Nottingham

${ }^{4}$ Department of Pediatrics, Faculty of Medicine, Dalhousie University, Halifax, Nova Scotia, Canada

${ }^{5}$ Department of Obstetrics and Gynaecology, Monash University, Clayton, Australia

Thank you for the opportunity to comment on the letter by Dr Xue from Shanghai Jiao Tong University. We agree there are many weaknesses in the data we reviewed. Dr Xue has identified one. Others are the incomplete reporting of infant feeding and mother-child interactions, and the frequent lack of infant testing to confirm or refute the possibility of vertical transmission of COVID-19. Finally, although we simply provided summary totals, it would be statistically preferable to combine series using the Mantel-Haenszel method and calculate a relative risk. We judged that doing this in light of the uncertainties around the data which Dr Xue has identified, might give a spurious precision to our results. As he says, more work is needed. For now we think it remains reasonable to not regard COVID-19 in itself, as an indication for Caesarean, artificial feeding or separation, in the mother and baby's interest. 